\title{
Quantized Feedback Control for Networked Control Systems Under Communication Constraints
}

\author{
Q.Q. Liu, G.H. Yang
}

\author{
Qing-Quan Liu \\ School of Information Science and Engineering \\ Shenyang Ligong University \\ No. 6, Nan Ping Zhong Road, Hun Nan Xin \\ District, Shenyang, 110159, China \\ E-mail: lqqneu@163.com \\ Guang-Hong Yang \\ College of Information Science and Engineering \\ Northeastern University \\ Shenyang, 110004, China
}

\begin{abstract}
This paper investigates the feedback stabilization problem for networked control systems (NCSs) with unbound process noise, where sensors and controllers are connected via noiseless digital channels carrying a finite number of bits per unit time. A sufficient condition for stabilization of NCSs, which relies on a variable-rate digital link used to transmit state measurements, is derived. A lower bound of data rates, above which there exists a quantization, coding and control scheme to guarantee both stabilization and a prescribed control performance of the unstable discrete-time plant, is presented. An illustrative example is given to demonstrate the effectiveness of the proposed method.
\end{abstract}

Keywords: networked control systems (NCSs), quantized feedback control, communication constraints, feedback stabilization.

\section{Introduction}

As is well known, in recent emerging applications (e.g., industrial automation, sensor networks, vehicle systems, aerospace industry and etc), the main aim is to control one or more dynamical systems employing multiple sensors and actuators transmitting and receiving information via a digital communication network. However, the limitations in the communication links often affect control performances significantly.

Our focus in this paper is on control under communication constraints. The research on the interplay among coding, estimation, and control was initiated by [1]. A high-water mark in the study of quantized feedback using data-rate limited feedback channels is known as the datarate theorem that states the larger the magnitude of the unstable poles, the larger the required data rate through the feedback loop. The intuitively appealing result was proved (see [2]- [5]), indicating that it quantifies a fundamental relationship between unstable physical systems and the rate at which information must be processed in order to stably control them. When the feedback channel capacity is near the data-rate limit, control designs typically exhibit chaotic instabilities. This result was generalized to different notions of stabilization and system models, and was also extended to multi-dimensional systems (see [6]- [8]). The research on Gaussian linear systems was addressed in [9]- [11]. Information theory was employed in control systems as a powerful conceptual aid, which extended existing fundamental limitations of feedback systems, and was used to derive necessary and sufficient conditions for robust stabilization of uncertain 
linear systems, Markov jump linear systems and unstructured uncertain systems (see [12]- [16]). The decentralized control schemes were addressed in [17]. The result on continuous-time linear Gaussian systems was derived in [18]. The result on time-varying communication channel was derived in [19]. Control under communication constraints inevitably suffers signal transmission delay, date packet dropout and measurement quantization which might be potential sources of instability and poor performance of control systems (see [22] and [23]). The survey papers [20] and [21] gave a historical and technical account of the various formulations.

The data-rate inequality is now well known and provides good intuition into the connection between achievable bit-rate and stability of linear time-invariant systems. It states that for a linear time-invariant system which is open-loop unstable, a controller can be designed to stabilize it if and only if the data rate $R$ around the closed feedback loop satisfies the data rate inequality: $R>\log |\operatorname{det}(A)|$ (bits/sample) where $A$ denotes the system matrix composed by only unstable modes. However, as the data rate $R$ is reduced to the critical value $\log |\operatorname{det}(A)|$, the plant states must always become unbounded. In engineering systems, It is of importance to present a lower bound of data rates above which there exists a quantization, coding and control scheme to guarantee both the stabilization and control performances of plants.

In this paper, we address quantized feedback control problem for stochastic linear systems with limited data rates. The aim is to present a lower bound of data rates above which there exists a coder-controller to guarantee both the stabilization and a prescribed control performance.

The following notation is adopted throughout this paper.

- Upper case variables, like $X$, represent random vectors.

- We write $\log _{2}(\cdot)$ simply as $\log (\cdot)$.

- Let $p(X)$ denote the probability density function of $X$ and $p(X \mid Y)$ denote the conditional probability density function of $X$ given $Y$.

- $\|\cdot\|$ represents either the Euclidean norm on a real vector space or the matrix norm induced by it.

- $E_{X}[\cdot]$ denotes expectation on $X$.

The remainder of this paper is organized as follows: Section II introduces problem formulation. Section III deals with quantized feedback control problem for NCSs with limited data rates. The results of numerical simulation are presented in Section IV. Conclusions are stated in Section V.

\section{Problem Formulation}

\subsection{Preliminaries}

We start this section by summarizing the main definitions of Information Theory and adopt [24], as a primary reference. The definitions listed in this section hold under general assumptions.

- Let $x$ and $y$ denote two random variables. The differential entropy $h(x)$ is defined as

$$
h(x):=E_{x}\left[\log \frac{1}{p(x)}\right] .
$$

The conditional differential entropy of $x$ given $y$ is defined as

$$
h(x \mid y):=E_{x, y}\left[\log \frac{1}{p(x \mid y)}\right] .
$$


- The mutual information between $x$ and $y$ is defined as

$$
I(x ; y):=h(x)-h(x \mid y)=E_{x, y}\left[\log \frac{p(x \mid y)}{p(x)}\right]
$$

- The information rate distortion function between $x$ and $y$ is defined as

$$
R(D):=\inf _{p(y \mid x) \in \Xi}\{I(x ; y)\}
$$

with $\Xi=\left\{p(y \mid x): E_{x, y}[d(x, y)] \leq D\right\}$. Therein, $d(x, y)$ denotes a distortion function or distortion measure, which is a mapping $d: x \times y \rightarrow \mathbb{R}^{+}$and $D$ is a given constant.

- Over communication channels, there are two different methods to define data rates. One is the code element rate $R_{c}(t)$ that denotes the number of code element transmitted in unit time, and the other is the information transmission rate $R_{e}(t)$ that denotes the amount of information transmitted in unit time. $R_{e}(t)$ may or may not be time-varying. We write $R_{e}(t)$ simply as $R(t)$. Therefore $R(t)$ is given by

$$
R(t)=\frac{1}{h} I(x ; y)(\mathrm{bits} / \mathrm{s})
$$

where $h$ is the transmission time for $I(x ; y)$.

In NCSs, after completing sample quantization procedure in state observation, we generally encode state values and transmit the information of plant states over digital channels. Therein, quantization precision and sampling period are determined by conditions for the stabilization of control systems and demands for control performances. Based on quantization precision and sampling period given, we may calculate the amount of information to be transmitted, which determine the value of the data rate $R(t)$. It means that the data rate $R(t)$ must satisfy requirements of the stabilization of control systems and control performances. obtain

Example 1. Assume $p(x)=\frac{1}{\sqrt{2 \pi \sigma_{1}^{2}}} e^{\frac{-x^{2}}{2 \sigma_{1}^{2}}}$ and $p(x \mid y)=\frac{1}{\sqrt{2 \pi \sigma_{2}^{2}}} e^{\frac{-x^{2}}{2 \sigma_{2}^{2}}}$. Let $h=1 s$. Then, we

$$
\begin{gathered}
h(x)=\frac{1}{2} \log 2 \pi e \sigma_{1}^{2} \text { (bits), } \\
h(x \mid y)=\frac{1}{2} \log 2 \pi e \sigma_{2}^{2} \text { (bits), } \\
I(x ; y)=\frac{1}{2} \log \frac{\sigma_{1}^{2}}{\sigma_{2}^{2}} \text { (bits). }
\end{gathered}
$$

Then, we quantity, encode the value $x$ and obtain the estimate $y$ of $x$. Here, $D=\sigma_{2}^{2}$. Thus, we have

$$
R(D)=\left\{\begin{array}{cc}
\frac{1}{2} \log \frac{\sigma_{1}^{2}}{\sigma_{2}^{2}} \text { (bits), } & 0 \leq \sigma_{2}^{2} \leq \sigma_{1}^{2} \\
0 \text { (bit), } & \sigma_{2}^{2}>\sigma_{1}^{2}
\end{array}\right.
$$

Finally, we transmit $y$, and the information transmission rate $R(t)$ must satisfy the following inequality:

$$
R(t) \geq \frac{1}{h} R(D)=\left\{\begin{array}{cc}
\frac{1}{2} \log \frac{\sigma_{1}^{2}}{\sigma_{2}^{2}}(\mathrm{bits} / \mathrm{s}), & 0 \leq \sigma_{2}^{2} \leq \sigma_{1}^{2}, \\
0(\mathrm{bit} / \mathrm{s}), & \sigma_{2}^{2}>\sigma_{1}^{2} .
\end{array}\right.
$$




\subsection{System Model}

Consider a stochastic linear system described by

$$
\begin{aligned}
& X(t+1)=A X(t)+B U(t)+F W(t), \\
& Y(t)=C X(t)
\end{aligned}
$$

where $X(t) \in \mathbb{R}^{n}$ is the state process, $U(t) \in \mathbb{R}^{m}$ is the control input, $Y(t) \in \mathbb{R}^{p}$ is the observation output, and $W(t) \in \mathbb{R}^{l}$ is the process disturbance. $A, B, C$ and $F$ are known constant matrices with appropriate dimensions (see Fig. 1).

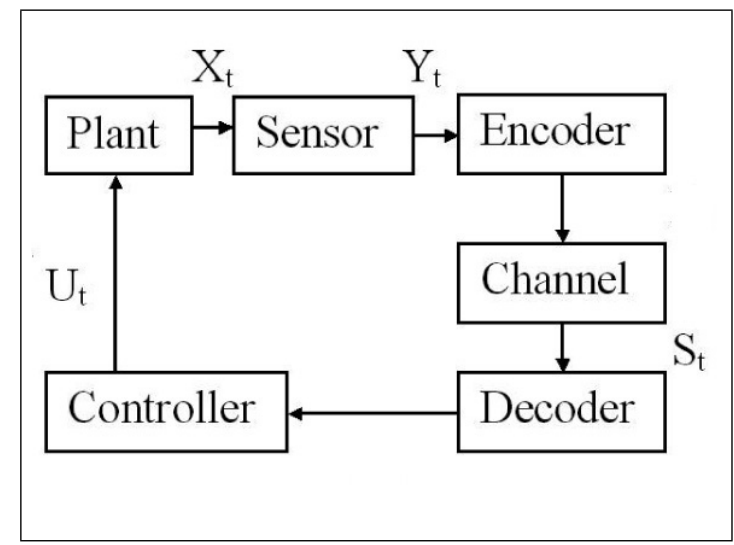

Figure 1: A networked control system with a communication channel.

The following is assumed to hold.

$A_{0}$. Without loss of generality, suppose that the pair $(A, B)$ is controllable, and the pair $(A, C)$ is observable.

$A_{1}$. The matrix $A$ is uniquely composed by unstable modes (having magnitude greater than or equal to unity).

$A_{2}$. The initial condition $X(0)$ and disturbance $W(0), \cdots, W(t)$ are mutually independent random variables with zero mean, satisfying $E\|X(0)\|_{2}^{2}<\Phi_{0}<\infty$ and $E\|W(t)\|_{2}^{2}<\Phi_{W}<\infty$, respectively.

$A_{3}$. The sensors and the controller are geographically separated and connected by errorless digital channels.

\subsection{Problem Statement}

We consider the system (1) under assumptions $A_{0}-A_{3}$ above. We quantify the performance of a coder-controller as a prescribed control performance by the asymptotic state norm

$$
J:=\lim _{t \rightarrow \infty} E\|X(t)\|^{2} .
$$

Here we are concerned with how small the plant states can be made as $t \rightarrow \infty$.

If the system (1) is controllable in the unstable modes, then there exists a matrix $K$ such that all eigenvalues of $A-B K$ have magnitude smaller than unity. In this case it seems logical to try to implement a quantized state feedback control law of the form $U(t)=-K \hat{X}(t)$ where $\hat{X}(t)$ denotes the estimate of $X(t)$. 
The main problem is to construct an encoder-decoder pair and to design a controller which guarantee both the stabilization and the control performance (2) of the system (1) in the mean square sense

$$
\lim _{t \rightarrow \infty} \sup E\|X(t)\|^{2}<\infty
$$

using the finite data rate provided by the digital feedback link.

\section{$3 \quad$ Feedback Control Under Data-Rate Constraints}

This section deals with the quantized feedback stabilization problem for the system (1). The purpose here is to present a quantization, coding and control scheme to stabilize the closed-loop system (1) with the performance (2) in the mean square sense (3). Assume that the encoder has access to the full state vector for practical plants. First we give a preliminary lemma.

Lemma $1^{[24]}$. Let $x \in \mathbb{R}$ denote a random variable and $\hat{x}$ denote an estimate of $x$. The expected distortion constraint is defined as $D \in \mathbb{R}^{+}$. For a given $D \geq E_{x}(x-\hat{x})^{2}$, there must exist a quantization and coding scheme if $R(t)$ satisfies

$$
R(t)>R(D) \geq \frac{1}{2} \log \frac{\sigma^{2}(x)}{D}(\text { bits } / \text { sample })
$$

where $\sigma^{2}(x)=E_{x}(x-E x)^{2}$.

Proof. See [24].

Remark 1.

- $R(D)$ denotes the lower bound of the amount of information which is needed to reconstruct the initial condition to some distortion fidelity which satisfies conditions for the stabilization. It means that more information available at the decoder will lead to better control performance.

- Since Lemma 1 gives a lower bound of data rate for all quantization and coding scheme, for a given $D, \hat{X}_{t}$ can be obtained by many quantization and coding schemes. However, $R(D)$ can not approach the fundamental lower limit if the scheme used is not optimal.

The following theorem is our main result in this paper.

Theorem 1. Consider the fully-observed system (1). Under assumptions $A_{0}-A_{3}$, given a state feedback control law of the form $U(t)=-K \hat{X}(t)$, satisfying that all eigenvalues of $A$-BK have magnitudes smaller than $1, \forall \varepsilon \in(0,1)$, the system (1) is mean-square stabilizable with

$$
J=\lim _{t \rightarrow \infty} E\|X(t)\|^{2}<\frac{1}{1-\varepsilon}\|F\|^{2} \Phi_{W}
$$

if the data rate $R(t)$ satisfies the following inequality:

$$
R(t)>\frac{1}{2} \log \frac{\left|\operatorname{det}\left[A^{T} A-(A-B K)^{T}(A-B K)\right]\right|}{\left|\operatorname{det}\left[\varepsilon I-(A-B K)^{T}(A-B K)\right]\right|} \text { (bits/sample). }
$$

Proof:

Consider the closed-loop system

$$
X(t+1)=A X(t)-B K \hat{X}(t)+F W(t)
$$

which we can also write as

$$
X(t+1)=A(X(t)-\hat{X}(t))+(A-B K) \hat{X}(t)+F W(t) .
$$


Then, we have

$$
\begin{aligned}
& E\|X(t+1)\|^{2} \\
& =\operatorname{trace}\left[E X(t+1) X^{T}(t+1)\right] \\
& \left.=\operatorname{trace}\left[A E(X(t)-\hat{X}(t))(X(t)-\hat{X}(t))^{T} A^{T}\right]+\operatorname{trace}\left[F E W(t) W^{T}(t) F^{T}\right]\right) \\
& +\operatorname{trace}\left[(A-B K) E \hat{X}(t) \hat{X}^{T}(t)(A-B K)^{T}\right]+2 \operatorname{trace}\left[(A-B K) E \hat{X}(t) W^{T}(t) F^{T}\right] \\
& +2 \operatorname{trace}\left[A E(X(t)-\hat{X}(t)) W^{T}(t) F^{T}\right]+2 \operatorname{trace}\left[A E(X(t)-\hat{X}(t)) \hat{X}^{T}(t)(A-B K)^{T}\right] .
\end{aligned}
$$

Notice that $\mathrm{X}(\mathrm{t})$ and $\mathrm{W}(\mathrm{t})$ are mutually independent. This implies

$$
\begin{gathered}
E \hat{X}(t) W^{T}(t)=0, \\
E(X(t)-\hat{X}(t)) W^{T}(t)=0
\end{gathered}
$$

following from assumption $A_{2}$. Furthermore, $E[X(t)-\hat{X}(t)] \hat{X}^{T}(t)=E V(t) \hat{X}^{T}(t)=0$ where $V(t)$ denotes the quantization error with zero mean. Substituting it and (5) into (4), we obtain

$$
E\|X(t+1)\|^{2}=\operatorname{trace} A \Sigma_{X(t) \mid \hat{X}(t)} A^{T}+\operatorname{trace}(A-B K) \Sigma_{\hat{X}(t)}(A-B K)^{T}+E\|F W(t)\|^{2}
$$

where we define $\Sigma_{X(t) \mid \hat{X}(t)}:=E(X(t)-\hat{X}(t))(X(t)-\hat{X}(t))^{T}$ and $\Sigma_{\hat{X}(t)}:=E \hat{X}(t) \hat{X}^{T}(t)$.

If assume that

$$
E\|X(t)\|^{2}>\operatorname{trace} A \Sigma_{X(t) \mid \hat{X}(t)} A^{T}+\operatorname{trace}(A-B K) \Sigma_{\hat{X}(t)}(A-B K)^{T}
$$

which is equivalent to

$$
\varepsilon E\|X(t)\|^{2}=\operatorname{trace}\left[A \Sigma_{X(t) \mid \hat{X}(t)} A^{T}\right]+\operatorname{trace}\left[(A-B K) \Sigma_{\hat{X}(t)}(A-B K)^{T}\right]
$$

with $\varepsilon \in(0,1)$, we see that

$$
E\|X(t+1)\|^{2}=\varepsilon E\|X(t)\|^{2}+E\|F W(t)\|^{2} .
$$

Then, we obtain

$$
\begin{aligned}
E\|X(t)\|^{2} & =\varepsilon^{t} E\|X(0)\|^{2}+\sum_{i=0}^{t-1} \varepsilon^{t-i-1} E\|F W(i)\|^{2} \\
& <\varepsilon^{t} \Phi_{0}+\frac{1-\varepsilon^{t}}{1-\varepsilon}\|F\|^{2} \Phi_{W}
\end{aligned}
$$

following from assumption $A_{2}$. Thus,

$$
J=\lim _{t \rightarrow \infty} E\|X(t)\|^{2}<\frac{1}{1-\varepsilon}\|F\|^{2} \Phi_{W} .
$$

It means that if we can design a quantization and coding scheme such that (6) holds, the system (1) is mean square stabilizable.

Next, we further argue that there exists a lower bound of the data rate $R(t)$ based on the quantization and coding scheme to stabilize the system (1) with

$$
J=\lim _{t \rightarrow \infty} E\|X(t)\|^{2}<\frac{1}{1-\varepsilon}\|F\|^{2} \Phi_{W} .
$$

Observe that

$$
\varepsilon E\|X(t)\|^{2}=\varepsilon \operatorname{trace} \Sigma_{X(t)}
$$


and

$$
\begin{aligned}
\Sigma_{\hat{X}(t)} & =E \hat{X}(t) \hat{X}^{T}(t)-E X(t) X^{T}(t)+E X(t) X^{T}(t) \\
& =\Sigma_{X(t)}-E\left(X(t) X^{T}(t)-\hat{X}(t) \hat{X}^{T}(t)\right) \\
& =\Sigma_{X(t)}-E(X(t)-\hat{X}(t))(X(t)-\hat{X}(t))^{T} \\
& =\Sigma_{X(t)}-\Sigma_{X(t) \mid \hat{X}(t)}
\end{aligned}
$$

where we define $\Sigma_{X(t)}:=E X(t) X^{T}(t)$. Substituting the equations above into (6), we have

$$
\begin{aligned}
\varepsilon \operatorname{trace} \Sigma_{X(t)}= & \operatorname{trace}\left[A \Sigma_{X(t) \mid \hat{X}(t)} A^{T}\right]+\operatorname{trace}\left[(A-B K) \Sigma_{\hat{X}(t)}(A-B K)^{T}\right] \\
= & \operatorname{trace}\left[A^{T} A \Sigma_{X(t) \mid \hat{X}(t)}\right]+\operatorname{trace}\left[(A-B K)^{T}(A-B K) \Sigma_{\hat{X}(t)}\right] \\
= & \operatorname{trace}\left[A^{T} A \Sigma_{X(t) \mid \hat{X}(t)}\right]+\operatorname{trace}\left[(A-B K)^{T}(A-B K)\left(\Sigma_{X(t)}-\Sigma_{X(t) \mid \hat{X}(t)}\right)\right] \\
= & \operatorname{trace}\left[\left(A^{T} A-(A-B K)^{T}(A-B K)\right) \Sigma_{X(t) \mid \hat{X}(t)}\right] \\
& +\operatorname{trace}\left[(A-B K)^{T}(A-B K) \Sigma_{X(t)}\right]
\end{aligned}
$$

which we can also write as

$$
\operatorname{trace}\left(\Theta_{1} \Sigma_{X(t)}\right)=\operatorname{trace}\left(\Theta_{2} \Sigma_{X(t) \mid \hat{X}(t)}\right)
$$

where we define $\Theta_{1}:=\varepsilon I-(A-B K)^{T}(A-B K)$ and $\Theta_{2}:=A^{T} A-(A-B K)^{T}(A-B K)$. Notice that there exist unitary matrices $P, Q, H$ that diagonalize the symmetric matrices $\Theta_{1}, \Theta_{2}, \Sigma_{X(t)}$ and $\Sigma_{X(t) \mid \hat{X}(t)}$, respectively. Namely, $\Theta_{1}=P^{T} \bar{\Theta}_{1} P, \Theta_{2}=Q^{T} \bar{\Theta}_{2} Q, \Sigma_{X(t)}=$ $H^{T} \Sigma_{\bar{X}(t)} H$ and $\Sigma_{X(t) \mid \hat{X}(t)}=H^{T} \Sigma_{\bar{X}(t) \mid \tilde{X}(t)} H$ where we define $\bar{X}(t):=H X(t), \tilde{X}(t):=H \hat{X}(t)$, $\bar{\Theta}_{1}:=\operatorname{diag}\left[\alpha_{1}, \cdots, \alpha_{n}\right]$ and $\bar{\Theta}_{2}:=\operatorname{diag}\left[\beta_{1}, \cdots, \beta_{n}\right]$. Then, (7) is equivalent to the following equations:

$$
\operatorname{trace}\left(P^{T} \Theta_{1} \Sigma_{X(t)} H\right)=\operatorname{trace}\left(Q^{T} \Theta_{2} \Sigma_{X(t) \mid \hat{X}(t)} H\right) .
$$

Since trace $M N=\operatorname{trace} N M$ with matrices $M \in R^{n \times m}$ and $N \in R^{m \times n}$ holds, thus the equality above is equivalent to

$$
\operatorname{trace}\left(\Sigma_{X(t)} H P^{T} \Theta_{1}\right)=\operatorname{trace}\left(\Sigma_{X(t) \mid \hat{X}(t)} H Q^{T} \Theta_{2}\right) .
$$

Then, we have

$$
\operatorname{trace}\left(H^{T} \Sigma_{X(t)} H P^{T} \Theta_{1} P\right)=\operatorname{trace}\left(H^{T} \Sigma_{X(t) \mid \hat{X}(t)} H Q^{T} \Theta_{2} Q\right)
$$

Namely

$$
\operatorname{trace}\left(\Sigma_{\bar{X}(t)} \bar{\Theta}_{1}\right)=\operatorname{trace}\left(\Sigma_{\bar{X}(t) \mid \tilde{X}(t)} \bar{\Theta}_{2}\right)
$$

Thus, we obtain

$$
\operatorname{trace}\left(\bar{\Theta}_{1} \Sigma_{\bar{X}(t)}\right)=\operatorname{trace}\left(\bar{\Theta}_{2} \Sigma_{\bar{X}(t) \mid \tilde{X}(t)}\right)
$$

which we can also write as

$$
\sum_{i=1}^{n} \alpha_{i} E \bar{x}_{i}^{2}(t)=\sum_{i=1}^{n} \beta_{i} E\left(\bar{x}_{i}(t)-\tilde{x}_{i}(t)\right)^{2}
$$

where define $\bar{X}(t):=\left[\bar{x}_{1}(t), \cdots, \bar{x}_{n}(t)\right]^{T}$ and $\tilde{X}(t):=\left[\tilde{x}_{1}(t), \cdots, \tilde{x}_{n}(t)\right]^{T}$. (8) can be viewed as a quantization and coding criterion. Then, based on the criterion (8), we can design a quantization and coding scheme to stabilize the system (1) in the mean square sense (3).

However, the analyses on the quantization and coding scheme rely heavily on the assumption of the boundability of the initial condition $\mathrm{X}(0)$ and the disturbance input $W(t)$. Similar to 
Shannon source coding, there exists a lower bound of the data rate $R(t)$, satisfying the criterion (8). We quantize each $\bar{x}_{i}(t)$ and obtain the estimate $\tilde{x}_{i}(t)$ of $\bar{x}_{i}(t)(i=1, \cdots, n)$, and encode it into a symbol $s_{t i}$ which is transmitted over an errorless digital channel. Then, the symbol $S_{t}=\left[s_{t 1}\right.$ $\left.\cdots s_{t n}\right]$ is decoded and converted into a control input $U_{t}=-B K \hat{X}_{t}=-B K H^{T} \tilde{X}_{t}$. Namely, we get $\hat{X}(t)=H^{T} \tilde{X}(t)$. Notice that $\sigma^{2}(\bar{x}) \leq E \bar{x}_{i}^{2}(t)$. Define $D:=\left[\begin{array}{lll}D_{1} \cdots D_{n}\end{array}\right]$. Thus, if let $D_{i} \leq \frac{\alpha_{i}}{\beta_{i}} \sigma^{2}\left(\bar{x}_{i}(t)\right)$, the criterion (8) must be satisfied. Following from Lemma 3.1, the following inequality can be obtained:

$$
\begin{aligned}
R_{i}(t) & >R_{i}\left(D_{i}\right) \\
& \geq \frac{1}{2} \log \frac{\sigma^{2}\left(\bar{x}_{i}(t)\right)}{D_{i}} \\
& \geq \frac{1}{2} \log \frac{\sigma^{2}\left(\bar{x}_{i}(t)\right)}{\frac{\alpha_{i}}{\beta_{i}} \sigma^{2}\left(\bar{x}_{i}(t)\right)} \\
& \geq \frac{1}{2} \log \frac{\beta_{i}}{\alpha_{i}} \text { (bits/sample). }
\end{aligned}
$$

Thus, it follows that

$$
\begin{aligned}
R(t) & =\sum_{i=1}^{n} R_{i}(t) \\
& >\frac{1}{2} \log \prod_{i=1}^{n} \frac{\beta_{i}}{\alpha_{i}} \\
& =\frac{1}{2} \log \frac{\left|\operatorname{det}\left(\bar{\Theta}_{2}\right)\right|}{\left|\operatorname{det}\left(\Theta_{1}\right)\right|} \\
& =\frac{1}{2} \log \frac{\left|\operatorname{det}\left(\Theta_{2}\right)\right|}{\left|\operatorname{det}\left(\Theta_{1}\right)\right|} \\
& =\frac{1}{2} \log \frac{\left|\operatorname{det}\left(A^{T} A-(A-B K)^{T}(A-B K)\right)\right|}{\left|\operatorname{det}\left(\varepsilon I-(A-B K)^{T}(A-B K)\right)\right|} \text { (bits/sample). }
\end{aligned}
$$

Thus, if $R(t)$ satisfies the inequality above, there exists a coder-controller to stabilize the system (1) with

$$
J=\lim _{t \rightarrow \infty} E\|X(t)\|^{2}<\frac{1}{1-\varepsilon}\|F\|^{2} \Phi_{W} .
$$

\section{Remark 2.}

- Theorem 1 states how fundamental tradeoffs between the data rate and the prescribed control performance. Clearly, the better performance can be achieve when the greater data rate is employed.

- Observe that corresponding to the classical situation without communication constraints, the performance becomes

$$
J=\lim _{t \rightarrow \infty} E\|X(t)\|^{2} \rightarrow\|F\|^{2} \Phi_{W}
$$

as the limit $R(t) \rightarrow \infty$.

\section{Numerical example}

To illustrate the effectiveness of communication constraints for stabilization of stochastic linear systems, we present an open loop unstable system as follows,

$$
X(t+1)=\left[\begin{array}{ccc}
1.61 & 0.52 & 0.25 \\
0.52 & 1.61 & 0.36 \\
-0.21 & 0.61 & 4.31
\end{array}\right] X(t)+\left[\begin{array}{l}
1 \\
1 \\
2
\end{array}\right] U(t)+0.3 W(t)
$$




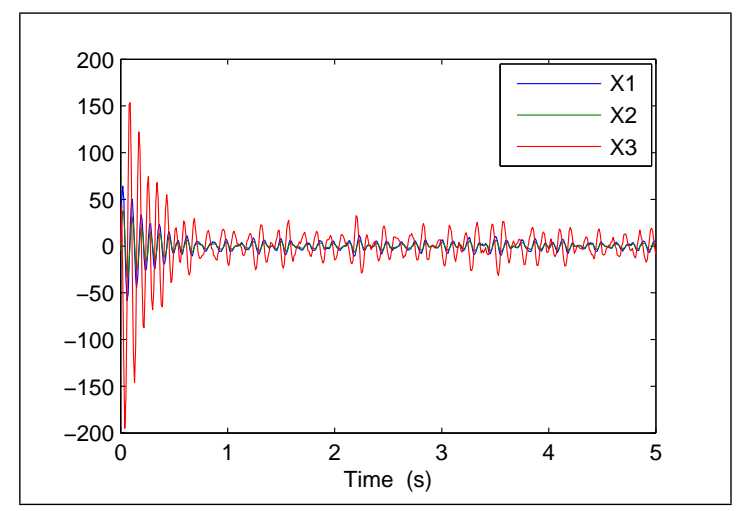

Figure 2: The system state responses with limited data rates.

Let $\varepsilon=0.4, \mathrm{X}(0)=\left[\begin{array}{lll}50 & -20 & -50\end{array}\right]^{T}$, and $\Phi_{W}=10$. Here we give a controller gain $K=$ [-0.6738 - 0.0681 2.9360]. A simulation is shown in Fig.2.

Remark 3. The obtained state responses are shown in Fig.2. It states that the encodingcontrol scheme based on Theorem 1 can stabilize the system above.

\section{Conclusion}

This paper addressed the feedback control problem for stochastic linear systems with limited data rates. The approach taken here was based on the hypothesis that sensors and controllers are connected by a rate-limited, time-varying communication channel. Based on the presented lower bound of data rates, there exists a coder-controller to guarantee both stabilization and a prescribed control performance. The simulation results have illustrated the effectiveness of the quantization, coding and control scheme.

\section{Acknowledgment}

This work was supported in part by the Funds for Creative Research Groups of China (No. 60821063), National 973 Program of China (Grant No. 2009CB320604), the Funds of National Science of China (Grant No. 60974043), the 111 Project (B08015), and the Funds of Doctoral Program of Ministry of Education, China (20100042110027).

\section{Bibliography}

[1] W. S. Wong and R. W. Brockett, Systems with finite communication bandwidth constraints II: Stabilization with limited information feedback, IEEE Trans. Automat. Control, 44(5):1049-1053, May 1999.

[2] J. Baillieul, Feedback designs for controlling device arrays with communication channel bandwidth constraints, in ARO Workshop on Smart Structures, Pennsylvania State Univ, Aug. 1999.

[3] J. Baillieul, Feedback designs in information based control, in Stochastic Theory and Control Proceedings of a Workshop Held in Lawrence, Kansas, B. Pasik-Duncan, Ed. New York: Springer-Verlag, 2001, pp. 35-57. 
[4] J. Baillieul, Data-rate requirements for nonlinear feedback control, in Proc. 6th IFAC Symp. Nonlinear Control Syst., Stuttgart, Germany, 2004, pp. 1277-1282.

[5] K. Li and J. Baillieul, Robust quantization for digital finite communication bandwidth (DFCB) control, IEEE Trans. Automat. Control, 49(9):1573-1584, Sep. 2004.

[6] G. N. Nair and R. J. Evans, Stabilizability of stochastic linear systems with finite feedback data rates, SIAM J. Control Optim., 43(2):413-436, Jul. 2004.

[7] N. Elia and S. K. Mitter, Stabilization of linear systems with limited information, IEEE Trans. Automat. Control, 46(9):1384-1400, Sep. 2001.

[8] N. Elia, When Bode meets Shannon: Control-oriented feedback communication schemes, IEEE Trans. Automat. Control, 49(9):1477-1488, Sep. 2004.

[9] S. Tatikonda and S. K. Mitter, Control under communication constraints, IEEE Trans. Automat. Control, 49(7):1056-1068, Jul. 2004.

[10] S. Tatikonda and S. K. Mitter, Control over noisy channels, IEEE Trans. Automat. Control, 49(7):1196-1201, Jul. 2004.

[11] S. Tatikonda, A. Sahai and S. K. Mitter, Stochastic linear control over a communication channel, IEEE Trans. Automat. Control, 49(9):1549-1561, Sep. 2004.

[12] N. C. Martins, M. A. Dahleh, and N. Elia, Feedback stabilization of uncertain systems in the presence of a direct link, IEEE Trans. Automat. Control, 51(3):438-447, Mar. 2006.

[13] N. C. Martins and M. A. Dahleh, Feedback control in the presence of noisy channels: 'Bodelike' fundamental limitations of performance, IEEE Trans. Automat. Control, 53(7):16041615, Jul. 2008.

[14] G. N. Nair, S. Dey, and R. J. Evans, Infimum data rates for stabilizing Markov jump linear systems, in Proc. IEEE Conf. Decision and Control, 2003, pp. 1176-1181.

[15] A. Sahai and S. Mitter, The necessity and sufficiency of anytime capacity for stabilization of a linear system over a noisy communication link Part I: Scalar systems, IEEE Trans. Automat. Control, 52(8):3369-3395, Aug. 2006.

[16] J. Q. Sun and S. M. Djouadi, Robust stabilization over communication channels in the presence of unstructured uncertainty, IEEE Trans. Automat. Control, 54(4):830-834, Apr. 2009.

[17] S. Yüksel and T. Basar, Communication constraints for decentralized stabilizability with time-invariant policies, IEEE Trans. Automat. Control, 52(6):1060-1066, Jun. 2007.

[18] C.D. Charalambous, A. Farhadi, and S.Z. Denic, Control of continuous-time linear Gaussian systems over additive Gaussian wireless fading channels: A separation principle, IEEE Trans. Automat. Control, 53(4):1013-1019, Apr. 2008.

[19] P. Minero, M. Franceschetti, S. Dey, and G. N. Nair, Data rate theorem for stabilization over time-varying feedback channels, IEEE Trans. Automat. Control, 54(2):243-255, Feb. 2009 .

[20] J. Baillieul and P. Antsaklis, Control and communication challanges in networked real time systems, in Proceedings of IEEE Special Iss. Emerg. Technol. Netw. Control Syst, USA: IEEE, 2007, pp. 9-28. 
[21] G. N. Nair, F. Fagnani, S. Zampieri, and R. J. Evans, Feedback control under data rate constraints: An overview, in Proceedings of IEEE Special Iss. Emerg. Technol. Netw. Control Syst, USA: IEEE, 2007, pp. 108-137.

[22] Y. L. Wang and G. H. Yang, $H_{\infty}$ control of networked control systems with time delay and packet disordering, IET Control Theory \&3 Applications, 1(5):1344-1354, May. 2007.

[23] Y. L. Wang and G. H. Yang, Multiple communication channels-based packet dropout compensation for networked control system, IET Control Theory $\mathscr{E}$ Applications, 2(8):717-727, Aug. 2008.

[24] T. Cover and J. Thomas, Elements of Information Theory. New York: Wiley, 2006. 